\title{
Multifocal osteonecrosis in a 3-year-old child with sickle beta plus thalassemia
}

\author{
Neha Bhasin ${ }^{1}$, Nathan Price ${ }^{1}$, and Sarah Desoky ${ }^{1}$ \\ ${ }^{1}$ University of Arizona Arizona Health Sciences Center
}

July 25, 2020

\begin{abstract}
Sickle beta + thalassemia $(\mathrm{S} \beta+)$ is considered to be a mild form of sickle cell disease (SCD). However, some patients with mild disease can present with osteonecrosis. Here, we present a three-year-old male who presented with acute pain and baseline hemoglobin of $13 \mathrm{~g} / \mathrm{dL}$ with multifocal osteonecrosis of multiple areas in his vertebrae, pelvis and femurs that improved with partial exchange transfusion and hydroxyurea.
\end{abstract}

\section{Case Description}

A 3-year-old Hispanic male with known sickle beta + thalassemia $\left(\mathrm{S} \beta^{+}\right)$presented to the hospital with severe lower abdomen and back pain. No recent fevers or chills. No recent travel, exposure to animals, or consumption of non-pasteurized foods. He had no prior history of vaso-occlusive pain crises (VOCs) but had two episodes of pneumonia since birth. No history of transfusions. Labs on admission are shown in Table 1. He received ibuprofen and morphine for pain. Abdominal ultrasound ruled out cholecystitis and intussusception. Magnetic resonance imaging (MRI) of his abdomen and pelvis with sedation was normal. Soon after the MRI, patient had a fever of 38.1 degree Celsius. Blood cultures were drawn and intravenous (IV) ceftriaxone was started. Patient was diagnosed with VOC and continued treatment with IV fluids, Toradol and morphine for pain management in inpatient hematology service. No further fevers occurred.

On day 4, he complained of left knee pain and refused to bear weight. On exam, his left knee pain was radiating from his hip. Initial plain films of the pelvis, left femur and left knee were normal. Blood cultures remained negative. C-reactive protein (CRP) was noted to be $46.6 \mathrm{mg} / \mathrm{L}(2-8 \mathrm{mg} / \mathrm{L})$. On day 6 , MRI of the hip and legs with and without contrast was performed and revealed multifocal osteonecrosis (OST) of proximal bilateral femurs, S2 vertebral body and bilateral ischium and ilium, and a small left hip effusion. (Figure 1A, B and C). ${ }^{1}$ Left hip effusion was aspirated by orthopedic surgery and showed no aerobic, anaerobic or fungal infections. No cytology could be done on this fluid. An echocardiogram showed no concerns for cardiac emboli. Coccidioides serologies showed IgM in indeterminate range and negative IgG. Subsequent serology 2 and 8 weeks later again showed indeterminate IgM and negative IgG making coccidioides unlikely. Patient continued to have pain but was able to be weaned to ibuprofen every 6 hours. Patient was discharged home with a slight limp with close follow-up with hematology and infectious disease service after 9 days of hospitalization. Discharge labs are shown in table 1.

On outpatient follow-up, he continued to be afebrile but had persistent limping, worse in the morning, despite taking ibuprofen every 6 hours. Rheumatology was consulted for concerns for autoimmune etiology of his MRI findings, such as chronic recurrent multifocal osteomyelitis (CRMO) or Juvenile Idiopathic Arthritis (JIA), which were both ruled out. Repeat MRI 2 months after presentation showed grossly unchanged findings from previous imaging. 
Given no other etiology of his multifocal OST other than sickle cell disease (SCD), we initiated concomitant hydroxyurea (HU) therapy at $20 \mathrm{mg} / \mathrm{kg} /$ day and partial exchange transfusions with the goal $\mathrm{HbS}$ of $<30 \%$. We aimed to continue this therapy for 6 months and assess clinical and imaging response. HU was maintained at the maximum tolerated doses. His lowest pre-transfusion HbS during this period was $39 \%$. His highest $\mathrm{HbF}$ level during this time was $15.5 \%$. Patient improved clinically with no residual pain or decreased range of motion by the end of the 6 month period. Repeat MRI of his hips and bilateral lower extremities after 6 months showed interval resolution of all previously described areas of OST. Some residual areas of signal abnormality were most prominent in the bilateral ilia and proximal femoral shafts (Figure 1D, E and F). No new signal abnormality or hip effusion was noted. Genetic testing was done that confirmed the diagnosis of $\mathrm{S} \beta^{+}$thalassemia with no concurrent $\alpha$-thalassemia trait.

Currently, our patient is being weaned off of partial exchange transfusions but remains on HU therapy and is being managed similar to HbSS and $\mathrm{HbS}^{-}$patients. ${ }^{2}$ We plan to assess his status regularly going forward and pursue repeat imaging 12 months later to insure resolution or stabilization of his previously noted multifocal OST.

\section{Discussion}

This case is a unique case of multifocal OST in a 3-year-old child with $\mathrm{S} \beta^{+}$thalassemia with a baseline $\mathrm{HbA}$ close to $20 \%$. This patient was noted to have significant acute OST of several bones in his lower body and lower vertebrae, something that has never been reported at this age in $\mathrm{S} \beta^{+}$thalassemia patients. The patient responded quite well to partial exchange transfusion therapy in addition to HU therapy.

The true incidence of OST and multifocal OST in $\mathrm{S} \beta^{+}$thalassemia is unknown. Normally, $\mathrm{S} \beta^{+}$thalassemia patients have $\mathrm{HbS}$ composition of approximately $65-90 \%$, $\mathrm{HbA} 5-30 \%$, $\mathrm{HbA} 2>3.5 \%$ and $\mathrm{HbF}$ of $2-10 \%$, similar to our patient. $\mathrm{S}^{+}$patients have mild anemia, occasional VOCs and OST. A recent case report showed a 40-year-old male with $\mathrm{S}^{+}{ }^{+}$with left femoral head OST who presented with fevers and chills in addition to back pain with a hemoglobin of $10.1 \mathrm{~g} / \mathrm{dL}$, a baseline $\mathrm{HbS}$ of $73.2 \%$ and $\mathrm{HbA}$ of $2.7 \%$, much lower than our patient who is only 3 years old. ${ }^{3}$

Known risk factors for OST in SCD include older age, male gender, high body mass index, leukopenia, HbSS with concurrent alpha-thalassemia trait (with relatively high hemoglobin, frequent VOEs, history of acute chest syndrome (ACS) and history of leg ulcers ${ }^{4-6}$ none of these applied to our patient. A recent database of $>6,200$ patients with SCD in California found an incidence of OST of $22 \%$ with no data available for $\mathrm{S}^{+}$thalassemia. ${ }^{4,5}$ A large prospective study of 2,524 patients looking at the incidence of OST of the humeral head in SCD showed no patients with $\mathrm{S} \beta^{+}$thalassemia younger than 25 years of age. ${ }^{7}$ In a prospective study of 200 patients with SCD, only 5 of 61 patients with OST had HbS $\beta$ thalassemia, however, no distinction was made between $\mathrm{HbS} \beta^{+}$and $\mathrm{HbS} \beta^{-}$thalassemia genotype in this study. ${ }^{8}$

OST of the femoral and humeral head is usually managed based on the Steinberg staging system. Patients with early disease can be managed conservatively with non-steroidal anti-inflammatory drugs (NSAIDs), hydration, crutch-weight bearing and sustained physical therapy. ${ }^{2}$ Core decompression can be the next step in patients where conservative measures do not work with arthroplasty as the final treatment. ${ }^{9,10}$ The role of HU and chronic transfusion therapy in SCD patients with OST for the treatment or prevention of OST is unknown. ${ }^{4,11}$

NSAID therapy was partially effective for our patient. However, given repeat imaging showing continued disease with motor compromise and no single surgical site to intervene, we tried partial exchange transfusion to decrease $\mathrm{HbS}$ with addition of $\mathrm{HU}$ therapy as a systemic treatment approach for treatment. Red cell exchange was recently reported as effective in a 37-year-old male with $\mathrm{HbS} \beta^{+}$thalassemia prior to surgery for multifocal OST. ${ }^{12}$ Transfusion has been shown to reduce the incidence of symptomatic AVN in children receiving regular transfusions to maintain $\mathrm{HbS}<30 \%$ and for prevention of recurrent stroke, however, there is no consensus on the use of transfusion for the sole purpose of preventing avascular necrosis. ${ }^{13}$ Future trials looking at this approach in patients with multifocal OST may be warranted based on our experience with this patient. 


\section{Conclusion}

Multifocal OST is uncommon in pediatric patients with $\mathrm{HbS} \beta^{+}$thalassemia. The role of transfusion therapy with or without the addition of hydroxyurea therapy in management of patients with multifocal OST needs further investigation.

\section{References}

1. Lee GC, Khoury V, Steinberg D, Kim W, Dalinka M, Steinberg M. How do radiologists evaluate osteonecrosis? Skeletal Radiol.2014;43(5):607-614.

2. Yawn BP, Buchanan GR, Afenyi-Annan AN, et al. Management of sickle cell disease: summary of the 2014 evidence-based report by expert panel members. JAMA. 2014;312(10):1033-1048.

3. Saxena S SN, Jaiswal RM. S-Beta Thalassemia leading to avascular necrosis of left hip joint in a young male - A rare case report.International Archives of Integrated Medicine. 2016;3(8):278-282.

4. Adesina OO, Neumayr LD. Osteonecrosis in sickle cell disease: an update on risk factors, diagnosis, and management. Hematology Am Soc Hematol Educ Program. 2019;2019(1):351-358.

5. Adesina O, Brunson A, Keegan THM, Wun T. Osteonecrosis of the femoral head in sickle cell disease: prevalence, comorbidities, and surgical outcomes in California. Blood Adv. 2017;1(16):1287-1295.

6. Hughes M, Akram Q, Rees DC, Jones AK. Haemoglobinopathies and the rheumatologist. Rheumatology (Oxford). 2016;55(12):2109-2118.

7. Milner PF, Kraus AP, Sebes JI, et al. Osteonecrosis of the humeral head in sickle cell disease. Clin Orthop Relat Res.1993(289):136-143.

8. Flouzat-Lachaniete CH, Roussignol X, Poignard A, Mukasa MM, Manicom O, Hernigou P. Multifocal joint osteonecrosis in sickle cell disease. Open Orthop J. 2009;3:32-35.

9. Pierce TP, Jauregui JJ, Elmallah RK, Lavernia CJ, Mont MA, Nace J. A current review of core decompression in the treatment of osteonecrosis of the femoral head. Curr Rev Musculoskelet Med.2015;8(3):228-232.

10. Issa K, Naziri Q, Maheshwari AV, Rasquinha VJ, Delanois RE, Mont MA. Excellent results and minimal complications of total hip arthroplasty in sickle cell hemoglobinopathy at mid-term follow-up using cementless prosthetic components. J Arthroplasty. 2013;28(9):1693-1698.

11. Howard J. Sickle cell disease: when and how to transfuse.Hematology Am Soc Hematol Educ Program. 2016;2016(1):625-631.

12. Mehra RA, Gupta SA, Borkar DB. Manual red cell exchange transfusion to avert sickle cell related complications. Asian J Transfus Sci.2018;12(2):157-159.

13. Davis BA, Allard S, Qureshi A, et al. Guidelines on red cell transfusion in sickle cell disease Part II: indications for transfusion. Br J Haematol. 2017;176(2):192-209.

\section{Hosted file}

Table 1-SB+.docx available at https://authorea.com/users/346066/articles/472159-multifocalosteonecrosis-in-a-3-year-old-child-with-sickle-beta-plus-thalassemia 
Figure 1: MRI Pre and Post partial exchange transfusion and hydroxyurea therapy
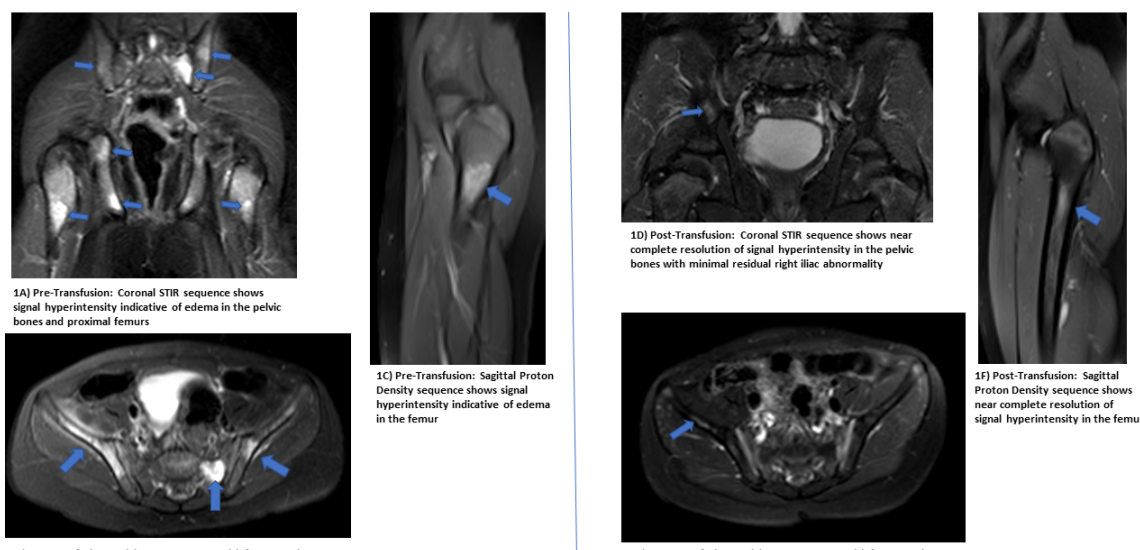

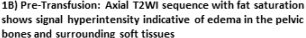

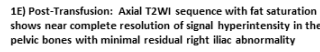

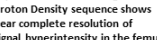

